\title{
Anomalous low-frequency noise in synthetic antiferromagnets: Possible evidence of current-induced domain-wall motion
}

\author{
D. Herranz, R. Guerrero, R. Villar, and F. G. Aliev \\ Departamento Física Materia Condensada, C-III, Universidad Autónoma de Madrid, Cantoblanco, Spain \\ and Instituto Nicolas Cabrera de Ciencia de Materiales, 28049 Madrid, Spain
}

A. C. Swaving and R. A. Duine

Institute for Theoretical Physics, Utrecht University, Leuvenlaan 4, 3584 CE, Utrecht, The Netherlands

C. van Haesendonck

Laboratory of Solid-State Physics and Magnetism, Katholieke Universiteit Leuven, Celestijnenlaan 200D, B3001 Leuven, Belgium

I. Vavra

IEE SAS, Dúbravská cesta 9, 84239 Bratislava, Slovakia

(Received 6 August 2008; revised manuscript received 6 March 2009; published 20 April 2009)

\begin{abstract}
We investigate current-driven magnetization dynamics in synthetic $[\mathrm{Fe} / \mathrm{Cr}]_{10}$ multilayer antiferromagnets by using low-frequency voltage noise measurements. We observe suppression of the noise above a critical current density of about $2 \times 10^{5} \mathrm{~A} / \mathrm{cm}^{2}$. Theoretical estimates suggest that this effect may be attributed to currentinduced motion of domain walls in the antiferromagnet. The observed critical current density is about one order of magnitude smaller than for ferromagnetic systems. Our results are relevant for applications of antiferromagnetic metal spintronics in, e.g., magnetic memory storage technology.
\end{abstract}

DOI: 10.1103/PhysRevB.79.134423

PACS number(s): 75.50.Ee, 72.25.Pn, 75.60.Ch

\section{INTRODUCTION}

The interaction between a collective coordinate, usually an order parameter that characterizes a phase transition, and nonequilibrium quasiparticles is common in physics. Examples are Andreev reflection of electronic quasiparticles of a superconductor and the influence of charge-density waves on electron transport. ${ }^{1}$ Prominent recent examples are giant magnetoresistance (GMR) (Refs. 2 and 3) and, in particular, spin transfer torques that are actively studied in the field of spintronics. ${ }^{4-7}$ Both effects occur due to the interaction of a spin-polarized current with the ferromagnetic order parameter. Currently, spin transfer is mostly studied in the context of current-driven domain-wall (DW) motion, which has been observed in ferromagnetic metals ${ }^{8-12}$ and ferromagnetic semiconductors. ${ }^{13-15}$

Recently, it was proposed theoretically that currentinduced torques also exist in antiferromagnets. ${ }^{16}$ Indirect evidence for their existence was found by the observation that the current influences the exchange bias in nanopillars. ${ }^{17,18}$ One of the most interesting aspects of current-induced torques in antiferromagnets is that the critical current for inducing magnetization dynamics is predicted to be substantially lower than in ferromagnetic systems. ${ }^{16}$ However, directly monitoring magnetization dynamics in antiferromagnets turns out to be hard because the resolution limit of the techniques commonly used to study ferromagnetic materials renders them unsuitable for studying antiferromagnets.

Noise measurements, on the other hand, have generally proven to be a powerful indirect diagnostic tool. For example, electronic shot noise has led to the observation of fractionally charged quasiparticles in fractional quantumHall systems. ${ }^{19}$ Very recently, a sliding charge-density wave was observed using low-frequency noise measurements. ${ }^{1,20}$

In this paper we report on current-driven magnetization dynamics in a synthetic antiferromagnet, observed via a change in the voltage noise as a function of current. We investigate the interaction of dc currents with domain walls in epitaxial antiferromagnetically coupled $[\mathrm{Fe} / \mathrm{Cr}]_{10}$ multilayers in a wide temperature range below $300 \mathrm{~K}$ and with current densities up to $5 \times 10^{5} \mathrm{~A} / \mathrm{cm}^{2}$, by using simultaneous measurements of domain-wall magnetoresistance and low-frequency noise. While the domain-wall magnetoresistance (DW-MR) remains practically unchanged for the current densities under investigation, a transition from excess DW-induced $1 / f$ noise at low current densities to a suppressed noise at current densities $j_{c}$ exceeding 2 $\times 10^{5} \mathrm{~A} / \mathrm{cm}^{2}$ is observed. We present theoretical estimates that indicate that this unexpected observation could be attributed to current-induced domain-wall motion above the critical current density $j_{c}$, which coarsens the DWs and reduces the DW-related noise. Current-induced DW noise in ferromagnetic Co line structures is found to remain qualitatively unchanged for densities up to $2 \times 10^{6} \mathrm{~A} / \mathrm{cm}^{2}$, indicating a weaker influence of current on DWs. We have chosen cobalt as a reference system and not $\mathrm{Fe} / \mathrm{Cr}$ multilayers with strongly suppressed antiferromagnetic coupling because Co is a fully ferromagnetic material. The $\mathrm{Fe} / \mathrm{Cr}$ multilayers might consist of two magnetic phases: the dominant one with local ferromagnetic order and a smaller antiferromagnetic fraction created by the unavoidable presence of monolayer steps in the Cr spacer.

As the observed critical current density for magnetization dynamics in the synthetic antiferromagnet is about one order of magnitude smaller than for ferromagnets, our results are important for applications of synthetic antiferromagnets in, 
e.g., memory storage technology. It should be noted that the domain structure and length scales of these materials are different from natural antiferromagnets. In the synthetic case the wavelength of the alternation of the magnetization direction is of the order of the $\mathrm{Fe} / \mathrm{Cr}$ layer thickness, whereas in a natural antiferromagnet this length scale would be atomic. Furthermore, the domain structure in the synthetic antiferromagnet is most likely induced by local variations in individual layer thickness. In a natural antiferromagnet the domain structure is caused by crystalline anisotropy (among other factors) which for example causes domain walls between different directions of antiferromagnetic ordering vectors. The current-in-plane of the constant magnetization is therefore hard to achieve with natural antiferromagnets. Nonetheless, we believe that our results present an important step in exploring spin torque effects beyond conventional ferromagnets.

\section{DOMAIN-WALL MAGNETORESISTANCE}

Two different epitaxial $[\mathrm{Fe}(001) / \mathrm{Cr}(001)]_{10}$ multilayers with the same $\mathrm{Cr}$ thickness of $13 \AA$ (providing the maximum antiferromagnetic coupling) and with different $\mathrm{Fe}$ thicknesses of $24 \AA$ [magnetic multilayer (MML1)] and of $12 \AA$ [magnetic multilayer (MML2)] were deposited in a molecular-beam epitaxy system on $\mathrm{MgO}(100)$ substrates held at $50{ }^{\circ} \mathrm{C}$. Detailed descriptions of the sample preparation and characterization [X-ray diffraction (XRD), electron transport, etc.] may be found in references of Aliev and Schad. ${ }^{21,22}$ The samples were patterned to a line structure configuration along the easy (001) axis with a length of $500 \mu \mathrm{m}$, a width of $10 \mu \mathrm{m}$, and a distance between pairs of oppositely situated voltage probes of $400 \mu \mathrm{m}$. Experiments were carried out with the dc current parallel to the external magnetic field and to the magnetically easy (001) axis. Details of the growth and characterization of 21-nm-thick, $10-\mu \mathrm{m}$-wide, and 700- $\mu \mathrm{m}$-long polycrystalline Co line structures can be found in references of Brems. ${ }^{23}$ The setup employed in electron transport and low-frequency noise measurements has been described previously. ${ }^{24,25}$

Creation of DWs in antiferromagnets is not energetically favorable. For synthetic antiferromagnets, such as $[\mathrm{Fe} / \mathrm{Cr}]_{n}$ MMLs, the influence of a magnetic field on the DWs is mainly due to the presence of biquadratic coupling, allowing effective displacement of the antiferromagnetic domain walls by the external fields. ${ }^{26}$ A relatively low DW pinning energy in fully epitaxial $\mathrm{Fe} / \mathrm{Cr} \mathrm{MMLs}^{27}$ gives rise to appreciable DW-MR only at temperatures below $100 \mathrm{~K}^{21}$ Although the physical origin of the low DW pinning barrier in the $\mathrm{Fe} / \mathrm{Cr}$ multilayers remains to be further clarified, we think that it might be related to the antiferromagnetic exchange coupling between Fe layers.

Magnetic force microscope (MFM) studies, which are sensitive to the perpendicular component of the local-field gradient and typically have resolution only down to $100 \mathrm{~nm}$, show a rather complicated character of the domains in our $[\mathrm{Fe} / \mathrm{Cr}]$ multilayers with irregular domain walls (see inset in Fig. 1). Our MFM images are qualitatively similar to those previously reported for $\mathrm{Fe} / \mathrm{Cr} / \mathrm{Fe}$ trilyers in the strongly an-

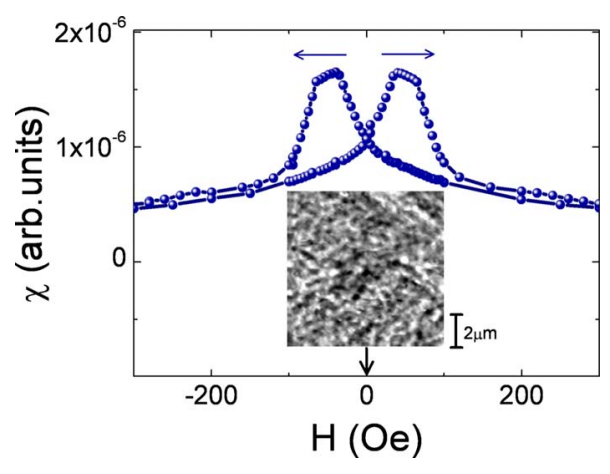

FIG. 1. (Color online) Main part: low-field dependence of the magnetic susceptibility in MML1 measured at $T=77 \mathrm{~K}$. The inset shows a MFM image of MML1 obtained in zero magnetic field at $T=300 \mathrm{~K}$ within $10 \times 10 \mu \mathrm{m}^{2}$.

tiferromagnetically coupled regime and measured at somewhat larger scales by using Kerr microscopy. ${ }^{28}$ We are not aware of any published micromagnetic calculations for $\mathrm{Fe} / \mathrm{Cr}$ or similar synthetic antiferromagnets which would allow comparison with these data. The main difficulty with such calculations is related to the rather small values of the demagnetizing fields (and corresponding energies to be minimized) in the synthetic antiferromagnets. Another factor contributing to the complicated domain structure present in the $\mathrm{Fe} / \mathrm{Cr}$ system will be discussed below in more detail and is related to the local crystal structure (one monolayer $\mathrm{Cr}$ steps) resulting in strong local variations of the magnetic coupling. ${ }^{29}$

The main part in Fig. 1 shows the magnetic-fielddependent susceptibility measured for MML1 along the easy axis and for the field and temperature range where domain walls contribute to the magnetoresistance. Although the large scale domain walls are created and annihilated at relatively low fields roughly below $100 \mathrm{Oe}$, the field-dependent magnetic susceptibility also reveals the presence of much smaller magnetic hysteresis above fields larger than 200 Oe. This feature is most probably related to gradual changes in the local-microstructure-induced magnetic disorder. More direct characterization of the changes in the local magnetic disorder up to saturation can, in principle, be provided by polarized neutron reflectometry (PNR) experiments. ${ }^{30,31}$ The magnetic multilayers studied in the present work are too thin to obtain appreciable PNR signals. On the other hand, increasing the total thickness of our epitaxial magnetic $\mathrm{Fe} / \mathrm{Cr}$ multilayers by increasing the number of magnetic layers (as employed in reference of Langridge ${ }^{30}$ for $\mathrm{Co} / \mathrm{Cu}$ MMLs) results in an enhancement of both roughness and microstructure related local magnetic disorder.

Figure 2(a) shows the low-temperature in-plane magnetoresistance in samples MML1 and MML2 measured up to high magnetic fields to ensure complete parallel alignment of the Fe layers. The high GMR values (60-80\%) point to the strong antiferromagnetic coupling and confirm the good epitaxy. We have found, however, that the saturation field $H_{s}$ is much better defined for MML1, indirectly indicating its enhanced crystalline order in comparison with MML2. In agreement with previous observations, both types of multilayers show an appreciable DW magnetoresistance only at temperatures below $100 \mathrm{~K}^{21,27}$ 

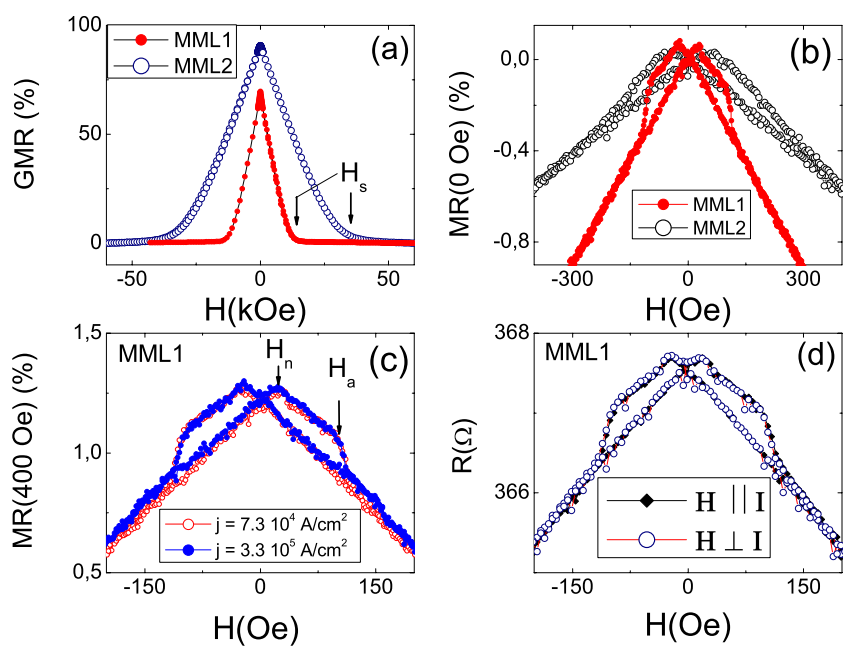

FIG. 2. (Color online) (a) Comparison of giant magnetoresistance $\mathrm{GMR}=[R(H)-R(60 \mathrm{kOe})] / R(60 \mathrm{kOe}) \times 100$ measured at $10 \mathrm{~K}$ for MML1 and MML2: The arrows indicate the corresponding saturation fields $H_{s}$. (b) Magnetoresistance $\mathrm{MR}(0 \mathrm{Oe})$ $=[R(H)-R(0 \mathrm{Oe})] / R(0 \mathrm{Oe}) \times 100$ for MML1 and MML2 measured at $77 \mathrm{~K}$. (c) Magnetoresistance $\mathrm{MR}(400 \mathrm{Oe})=[R(H)$ $-R(400 \mathrm{Oe})] / R(400 \mathrm{Oe}) \times 100$ for MML1 measured at $T=77 \mathrm{~K}$ with different current densities that were also used for the noise measurements. The arrows indicate the DW nucleation $\left(H_{n}\right)$ and annihilation $\left(H_{a}\right)$ fields. (d) Low-field magnetoresistance for MML1 measured at $T=77 \mathrm{~K}$ with very small current density and with current either parallel or perpendicular to the external magnetic field.

Figure 2(b) compares the normalized magnetoresistance, measured at a temperature of $77 \mathrm{~K}$ in MML1 and MML2, for the low magnetic field regions where DWs nucleated at $H$ $=H_{n}$ and annihilated at $H=H_{a}$ contribute to magnetoresistance. One clearly observes a less-defined DW annihilation field for MML2 when compared to MML1, which correlates with a much broader field region needed to suppress completely the antiferromagnetic state [Figs. 2(a) and 2(b)]. This further indicates the presence of higher local structural order in MML1 when compared to MML2.

We have found that the DW-MR is independent of the applied current density up to the maximum applied current densities. Figure 2(c) demonstrates that even with the maximum current density used at $T=77 \mathrm{~K} \quad(j=3.3$ $\times 10^{5} \mathrm{~A} / \mathrm{cm}^{2}$ ), the current-induced self-field or heating are negligible and practically have no influence on the DW-MR. To finalize our discussion of the low-field electron transport we also mention that the DW-MR is found to be practically independent of orientation of the current with respect to the in-plane magnetic field [see Fig. 2(d)]. This clearly indicates the absence of anisotropic magnetoresistance, as expected for strongly antiferromagnetically coupled multilayers with reduced magnetization.

\section{NOISE MEASUREMENTS}

The voltage noise power $S_{V}$ due to magnetization fluctuations was studied in the frequency range between 1 and 40 Hz. The observed $1 / f$ noise can be expressed in terms of

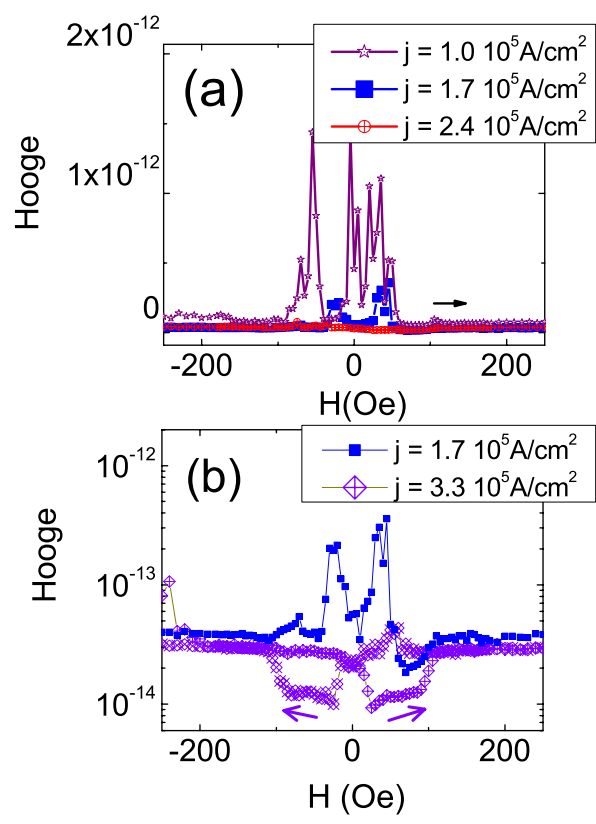

FIG. 3. (Color online) (a) Dependence of the Hooge factor $(\alpha)$ on the magnetic field for MML1 at $77 \mathrm{~K}$ with different applied current densities. (b) Hooge factor plotted on a logarithmic scale vs magnetic field for MML1 at $77 \mathrm{~K}$ with two different applied current densities. The normalized noise vs field measurements are done with a current density of $j=3.3 \times 10^{5} \mathrm{~A} / \mathrm{cm}^{2}$ and the results are shown both for increasing and decreasing magnetic field. The experiments have been carried out with dc current parallel to the external magnetic field and to the magnetic easy (001) axis.

$S_{V}(f, H)=\frac{\alpha(H) V^{2}}{f}$, with $\alpha$ the Hooge factor and $V$ the average voltage between potential contacts. ${ }^{32,33}$ Figures 3 and 4 present our main finding: there occurs a clear suppression of the DW-related excess low-frequency noise (Hooge factor) in MML1 for sufficiently high current densities. Figures 3(a) and 3(b) show data obtained at $T=77 \mathrm{~K}$ when the current density exceeds $2 \times 10^{5} \mathrm{~A} / \mathrm{cm}^{2}$, the excess noise observed for low magnetic fields, where DWs are created and propagate, is suppressed in the field interval $H_{n}<H<H_{a}$. Measurements under similar conditions $(77 \mathrm{~K})$ for MML2 reveal that the Hooge factor is about an order of magnitude smaller and the noise shows no qualitative changes up to current densities of $5 \times 10^{5} \mathrm{~A} / \mathrm{cm}^{2}$ (not shown). This provides further proof for stronger DW pinning due to higher structural disorder in MML2.

Figures 4(a) and 4(b) show direct voltage noise power measurements for MML1 presented for two limiting cases: small vs high current density with the magnetic field either inside or outside the interval $\left(H_{n}<H<H_{a}\right)$ where antiferromagnetic DWs nucleate and propagate. Figure 4(c) demonstrates the relatively good scaling of the averaged, over the frequencies of interest $(1-25 \mathrm{~Hz})$, noise power by the frequency with the square of bias voltage at $77 \mathrm{~K}$, which is expected for the phenomenological Hooge law describing near equilibrium $1 / f$ noise. For the weak field $H_{n}<H<H_{a}$ and weak bias interval, however, a strong deviation from the above dependence is observed. We attribute the excess noise and deviation from the $1 / f$-like frequency dependence to nonequilibrium noise present at low fields $\left(H_{n}<H<H_{a}\right)$ to a 


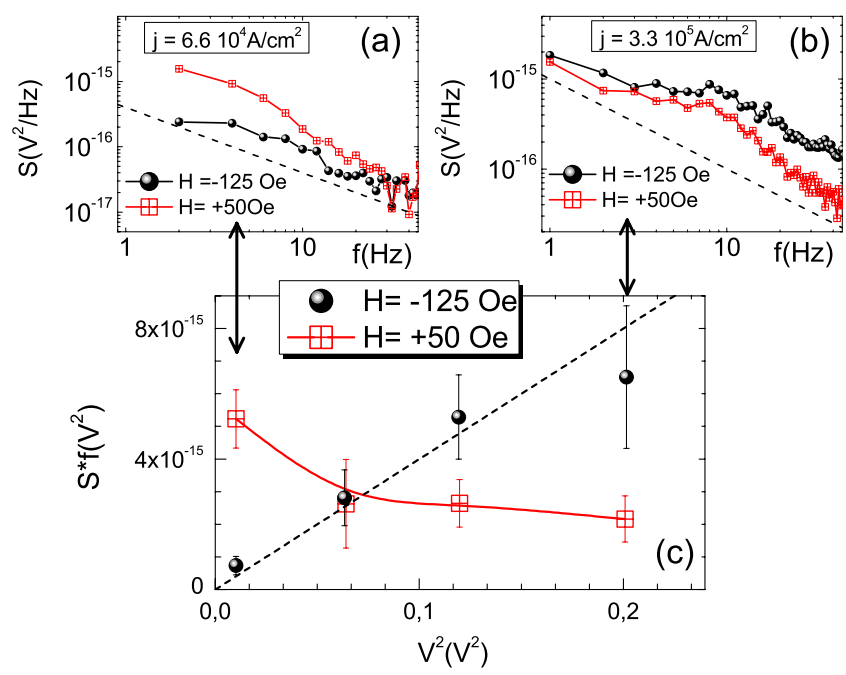

FIG. 4. (Color online) [(a) and (b)] Voltage noise (power spectral density) for MML1 for low and high biases, $j=6.6$ $\times 10^{4} \mathrm{~A} / \mathrm{cm}^{2}$ and $j=3.3 \times 10^{5} \mathrm{~A} / \mathrm{cm}^{2}$, respectively, for different applied fields inside $(H=+50 \mathrm{Oe})$ or outside $(H=-125 \mathrm{Oe})$ the interval $\left(H_{n}<H<H_{a}\right)$ where antiferromagnetic DWs nucleate and propagate at $77 \mathrm{~K}$ : The dotted lines represent $1 / f$ variation in the noise power, while the black arrows relate the applied bias with the current density. Part (c) plots the averaged over the frequencies $(1-25 \mathrm{~Hz})$ product of the power spectral density multiplied by the frequency as a function of square of the applied bias voltage $\mathrm{V}^{2}$. A nearly linear dependence $S_{V} \times f=\alpha(H) V^{2}$ is seen for the field $H=$ -125 Oe outside the interval where antiferromagnetic DWs nucleate and propagate, whereas in the field range $\left(H_{n}<H<H_{a}\right)$ the parameter $S_{V} \times f$ exhibits a clearly different behavior discussed in the text.

Barkhausen-type contribution to the noise at low biases [Figs. 4(c)]. Despite this deviation, our work uses the Hooge factor as a simplified parameter for the analysis of the normalized noise.

Decreasing the temperature down to $10 \mathrm{~K}$ substantially reduces the excess DW-induced noise measured in MML1 for low current densities [Fig. 5(a)]. This is in perfect agreement with the suppression of the DW-related excess imaginary contribution to the magnetic susceptibility due to stronger DW pinning at low temperatures. ${ }^{26}$ The noise measured at $10 \mathrm{~K}$ with low current density $\left(<10^{5} \mathrm{~A} / \mathrm{cm}^{2}\right)$ weakly increases when DWs are formed. However, for current densities exceeding $2 \times 10^{5} \mathrm{~A} / \mathrm{cm}^{2}$ a strong reduction in the normalized noise for the field region where DWs are created and propagate is again observed. Figures 5(b) and 5(c) compare the dependence of the Hooge factor on current density for the field regions with $\left(H_{n}<H<H_{a}\right)$ and without $\left(H>H_{a}\right)$ the presence of DWs. The crossover from excess to suppressed DW is shifted to lower current densities for $T=10 \mathrm{~K}$ when compared to $T=77 \mathrm{~K}$. We believe that part of the currentinduced suppression of the DW-related noise at the highest current densities and low temperatures [Fig. 5(c)] may be due to Joule heating and Oersted field effects that are estimated to be about $10 \mathrm{~K}$ and 5 Oe for a current density of $4 \times 10^{5} \mathrm{~A} / \mathrm{cm}^{2}$. We finally mention that qualitatively similar current-induced changes in the normalized low-frequency noise have been observed when experiments have been car-
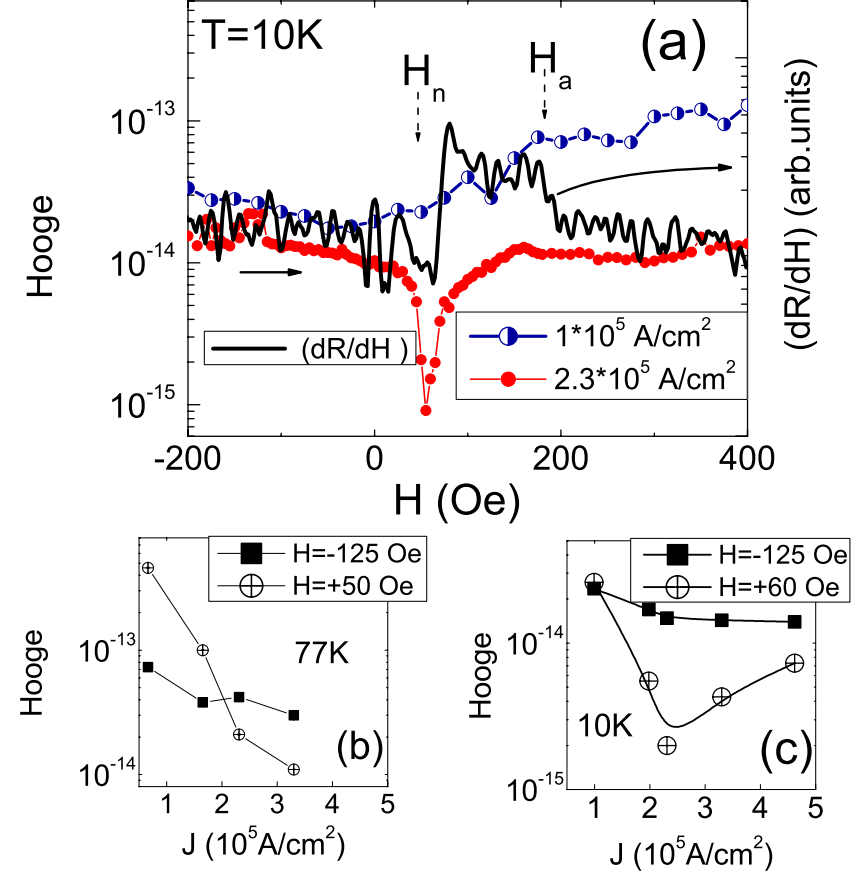

FIG. 5. (Color online) (a) Dependence of the Hooge factor $(\alpha)$ (on logarithmic scale) on the magnetic field for MML1 obtained from noise measurements at $T=10 \mathrm{~K}$ with two different applied current densities. For comparison, the solid line shows the absolute values of the derivative $\partial R / \partial H$ measured with a current density of $2.3 \times 10^{5} \mathrm{~A} / \mathrm{cm}^{2}$. The vertical arrows mark the regions of nucleation and annihilation of the domain walls determined from lowfield magnetoresistance measurements. [(b) and (c)] Dependence of the Hooge factor on the current density measured in MML1 with and without domain walls for two different magnetic fields at temperatures of $77 \mathrm{~K}$ (part b) and of $10 \mathrm{~K}$ (part c).

ried out with the dc current along the easy axis, but perpendicular to the external magnetic field.

It was recently reported that the DW-induced noise measured within the field region where DWs are formed, propagate, and annihilate under quasiequilibrium conditions, approximately scales with the absolute values of the derivative of resistance vs magnetic field, i.e., $\alpha(H) \propto \frac{\partial R}{\partial H} .{ }^{34}$ Figure 5(a) compares the field dependence of $\partial R / \partial H$ and of the Hooge factor measured at $10 \mathrm{~K}$ with a current density of 2.3 $\times 10^{5} \mathrm{~A} / \mathrm{cm}^{2}$. Clearly, the Hooge factor and $\partial R / \partial H$ reveal qualitatively different field dependences close to the region where DWs are formed and propagate. This observation indicates the presence of strongly nonequilibrium DW induced noise at high current densities and also is an indirect proof of current-induced motion of the DWs in Fe/Cr multilayers. Interestingly, we have found that the simple relation between $\partial R / \partial H$ and the Hooge factor is reasonably well fulfilled for our noise measurements in the ferromagnetic Co line structure in the field region where DWs are nucleated and propagate up to current densities of about $4 \times 10^{6} \mathrm{~A} / \mathrm{cm}^{2}$ (Fig. 6). Qualitatively similar behavior was observed both for 300 and $77 \mathrm{~K}$. For current densities exceeding $10^{6} \mathrm{~A} / \mathrm{cm}^{2}$ the Oersted field created by the transport current (roughly 12 Oe for the maximum used current density) and Joule heating (being above $10 \mathrm{~K}$ for the maximum applied dc currents) may, how- 


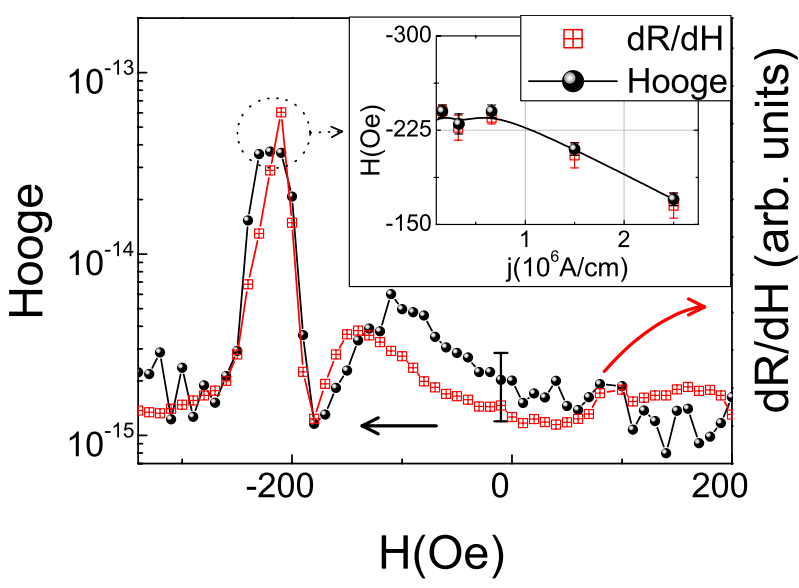

FIG. 6. (Color online) Hooge factor $(\alpha)$ vs magnetic field measured in the ferromagnetic Co line structure at $77 \mathrm{~K}$ with a current density of $3.3 \times 10^{5} \mathrm{~A} / \mathrm{cm}^{2}$. The inset shows the field values of the maximum in the Hooge factor and the related maxima of the derivative $\partial R / \partial H$ as a function of current density.

ever, affect both magnetotransport $(\partial R / \partial H)$ and lowfrequency noise in the ferromagnetic Co line structures. This influence is evidenced in the gradual reduction in the domain-wall depinning field and related displacement of both maxima in both $\partial R / \partial H$ and the Hooge factor above $10^{6} \mathrm{~A} / \mathrm{cm}^{2}$ (see inset to Fig. 6).

\section{DISCUSSION AND CONCLUSIONS}

We believe our experimental results are consistent with current-induced motion of the domains in the magnetic microstructure in the $\mathrm{Fe} / \mathrm{Cr}$ multilayers, as we now explain. First of all, the predominant domain walls are those in the $\mathrm{Fe}$ layers induced by local variation of the Cr layer thickness, which causes alternating exchange coupling between the $\mathrm{Fe}$ layers ${ }^{29}$ as well as extrinsic pinning for the domain walls. Second, because the geometry is current-in-plane of the layers, we assume that within each layer the same mechanisms prevail that cause current-induced domain-wall motion in ferromagnetic metallic wires. In the presence of extrinsic pinning the critical current for depinning a domain wall is then given by ${ }^{35,36} j_{c} \sim|e| V_{\operatorname{pin}} \lambda /(\hbar \beta A \xi)$, where $e$ is the electron charge, $V_{\text {pin }}$ is a typical pinning energy for the domain wall, and $A$ is the cross-sectional area perpendicular to the current direction of a single Fe layer. For our system parameters we find, taking $V_{\text {pin }} / k_{B}$ equal to the temperature below which DW magnetoresistance is observed $(\sim 100 \mathrm{~K})$, that $j_{c} \sim \lambda / \beta \xi \times 10^{5} \mathrm{~A} / \mathrm{cm}^{2}$ in terms of the width $\lambda$ of the domain wall and the typical range $\xi$ of the pinning potential. The critical current is further determined by the dimensionless parameter $\beta$ that characterizes the degree to which spin is not conserved in the spin transfer process, and/or the degree to which the spin transfer torque is not adiabatic. In our system we expect that $\lambda / \xi$ is of the order of $0.1-0.01 .^{29}$ Microscopic calculations indicate that $\beta$ is of the same order as, though generally not precisely equal to, the Gilbert damping constant $(\sim 0.1-0.01)$ (Refs. 37-40) and we conclude that the estimated critical current is consistent with our ex- perimental findings. From this theoretical picture we also conclude that the critical current for moving domain walls is lower in multilayer $\mathrm{Fe} / \mathrm{Cr}$ than in ferromagnetic wires, mainly because the pinning energy of the domain walls is smaller due to the antiferromagnetic structure. To further corroborate the picture of extrinsic pinning, we note that estimating the so-called intrinsic critical current for domain-wall motion from the saturation magnetization of one $\mathrm{Fe}$ layer $(\sim 2 T)$ yields a critical current that is several orders of magnitude higher than the current at which the suppression of $1 / f$ noise is observed. ${ }^{35}$

Let us finally discuss the possible origin of the unusual minimum in the normalized $1 / f$ noise at high current densities and for magnetic fields in between the DW nucleation and depinning fields. When current-induced torques overcome the DW pinning energy and for high current densities the DWs are set in motion, their continuous displacement could essentially reduce a local variation in the magnetic coupling sign due to $1 \mathrm{ML}$ steps at the $\mathrm{Fe} / \mathrm{Cr}$ interface previously reported for $\mathrm{Fe} / \mathrm{Cr} / \mathrm{Fe}$ multilayers. ${ }^{29}$ The presence of interfacial roughness induced magnetic disorder in $[\mathrm{Fe} / \mathrm{Cr}]_{n}$ multilayers with large $n=30-80$, which is influenced by high (close to saturation) magnetic fields, was also identified by using neutron off-specular diffusive scattering experiments. ${ }^{31}$ Here, we suggest that the substantial suppression of magnetic noise in the vicinity of the depinning field with high current densities $j_{c}>2 \times 10^{5} \mathrm{~A} / \mathrm{cm}^{2}$ is due to large DW movement induced reduction in the local magnetic disorder. In other words, we propose that the downward and upward variations in the magnetic noise at low fields appears because of competition between local magnetic disorder (which provides the background magnetic noise) and its reduction by the moving domain walls due to larger currents. We note that our hypothesis is also corroborated by the observation of broad maxima in the noise at low temperatures $(T=10 \mathrm{~K})$ and at low current densities [Fig. 5(c)]. We believe that the weakly mobile domains at low currents could contribute to the enhanced low-field magnetic noise while the DWs moving by the current suppress the local microstructure induced magnetic disorder and the related low-frequency noise.

In conclusion, detailed studies of domain-wall magnetoresistance and noise in antiferromagnetically coupled $\mathrm{Fe} / \mathrm{Cr}$ multilayers reveal an unexpected dependence of domainwall-induced low-frequency noise on the current density. We attribute this observation to current-induced magnetization dynamics that occurs at a critical current density that is smaller than typical current densities observed in ferromagnetic materials.

\section{ACKNOWLEDGMENTS}

It is a great pleasure to acknowledge Allan MacDonald and Gen Tatara for helpful conversations. We also thank Alexander Volodin for taking the MFM image and Vasilij Smatko for lithography. The work was supported by Comunidad de Madrid (Grant No. S-505/MAT0194), Spanish MEC (Grant No. MAT2006-07196, Consolider-Nanociencia 
Molecular Grant No. CSD2007-00010), and the Nederlandse Organisatie voor Wetenschaplijk Onderzoek $(N W O)$. This work, as a part of the European Science Foundation EUROCORES Programme (Grant No. 05-FONE-FP-010-
SPINTRA), was also supported by funds from the Spanish MEC (Grant No. MAT2006-28183-E), and the EC Sixth Framework Programme (Contract No. ERAS-CT-2003980409).
${ }^{1}$ S. Cox, J. Singleton, R. D. McDonald, A. Migliori, and P. B. Littlewood, Nature Mater. 7, 25 (2008).

${ }^{2}$ M. N. Baibich, J. M. Broto, A. Fert, F. Nguyen Van Dau, F. Petroff, P. Etienne, G. Creuzet, A. Friederich, and J. Chazelas, Phys. Rev. Lett. 61, 2472 (1988).

${ }^{3}$ G. Binasch, P. Grunberg, F. Saurenbach, and W. Zinn, Phys. Rev. B 39, 4828 (1989).

${ }^{4}$ M. Tsoi, A. G. M. Jansen, J. Bass, W. C. Chiang, M. Seck, V. Tsoi, and P. Wyder, Phys. Rev. Lett. 80, 4281 (1998).

${ }^{5}$ E. B. Myers, D. C. Ralph, J. A. Katine, R. N. Louie, and R. A. Buhrman, Science 285, 867 (1999).

${ }^{6}$ J. C. Slonczewski, J. Magn. Magn. Mater. 159, L1 (1996).

${ }^{7}$ L. Berger, J. Appl. Phys. 81, 4880 (1997).

${ }^{8}$ J. Grollier, P. Boulenc, V. Cros, A. Hamzić, A. Vaurès, A. Fert, and G. Faini, Appl. Phys. Lett. 83, 509 (2003).

${ }^{9}$ N. Vernier, D. A. Allwood, D. Atkinson, M. D. Cooke, and R. P. Cowburn, Europhys. Lett. 65, 526 (2004).

${ }^{10}$ A. Yamaguchi, T. Ono, S. Nasu, K. Miyake, K. Mibu, and T. Shinjo, Phys. Rev. Lett. 92, 077205 (2004).

${ }^{11}$ M. Kläui, C. A. F. Vaz, J. A. C. Bland, W. Wernsdorfer, G. Faini, E. Cambril, L. J. Heyderman, F. Nolting, and U. Rüdiger, Phys. Rev. Lett. 94, 106601 (2005).

${ }^{12}$ D. Ravelosona, D. Lacour, J. A. Katine, B. D. Terris, and C. Chappert, Phys. Rev. Lett. 95, 117203 (2005).

${ }^{13}$ Y. Jiang, T. Nozaki, S. Abe, T. Ochiai, A. Hirihata, N. Tezuka, and K. Inomata, Nature Mater. 3, 363 (2004).

${ }^{14}$ S. Mangin, D. Ravelosona, J. Katine, M. Carey, B. Terris, and E. Fullerton, Nature Mater. 5, 210 (2006).

${ }^{15}$ M. Yamanouchi, D. Chiba, F. Matsukura, and H. Ohno, Nature (London) 428, 539 (2004).

${ }^{16}$ A. S. Nunez, R. A. Duine, P. Haney, and A. H. MacDonald, Phys. Rev. B 73, 214426 (2006).

${ }^{17}$ Z. Wei, A. Sharma, A. S. Nunez, P. M. Haney, R. A. Duine, J. Bass, A. H. MacDonald, and M. Tsoi, Phys. Rev. Lett. 98, 116603 (2007).

${ }^{18}$ S. Urazhdin and N. Anthony, Phys. Rev. Lett. 99, 046602 (2007).

${ }^{19}$ L. Saminadayar, D. C. Glattli, Y. Jin, and B. Etienne, Phys. Rev. Lett. 79, 2526 (1997).

${ }^{20}$ E. R. Nowak, Physica C 421, 15 (2005).

${ }^{21}$ F. Aliev, R. Schad, A. Volodin, C. van Haesendock, Y. Bruynseraede, I. Vavra, V. Dugaev, and R. Villar, Europhys. Lett. 63, 888
(2003).

${ }^{22}$ R. Schad, P. Belien, G. Verbanck, V. V. Moshchalkov, Y. Bruynseraede, H. E. Fischer, S. Lefebvre, and M. Bessiere, Phys. Rev. B 59, 1242 (1999).

${ }^{23}$ S. Brems, D. Buntinx, K. Temst, C. Van Haesendonck, F. Radu, and H. Zabel, Phys. Rev. Lett. 95, 157202 (2005).

${ }^{24}$ R. Guerrero, F. Aliev, R. Villar, J. Hauch, M. Fraune, G. Gntherodt, K. Rott, H. Bruckl, and G. Reiss, Appl. Phys. Lett. 87, 042501 (2005).

${ }^{25}$ R. Guerrero, F. G. Aliev, Y. Tserkovnyak, T. S. Santos, and J. S. Moodera, Phys. Rev. Lett. 97, 266602 (2006).

${ }^{26}$ F. G. Aliev, J. L. Martinez, V. V. Moshchalkov, Y. Bruynseraede, A. P. Levanyuk, and R. Villar, Phys. Rev. Lett. 88, 187201 (2002).

${ }^{27}$ F. Aliev, R. Villar, R. Schad, and J. Martinez, Mater. Res. Soc. Symp. Proc. 746, 81 (2003).

${ }^{28}$ M. Rührig, R. Schäfer, A. Hubert, R. Mosler, J. A. Wolf, S. Demokritov, and P. Grünberg, Phys. Status Solidi A 125, 635 (1991).

${ }^{29}$ C. M. Schmidt, D. E. Burgler, D. M. Schaller, F. Meisinger, and H. J. Guntherodt, Phys. Rev. B 60, 4158 (1999).

${ }^{30}$ S. Langridge, J. Schmalian, C. H. Marrows, D. T. Dekadjevi, and B. J. Hickey, Phys. Rev. Lett. 85, 4964 (2000).

${ }^{31}$ Masayasu Takeda, Y. Endoh, A. Kamijo, and J. Mizuki, Physica B 248, 14 (1998).

${ }^{32}$ H. T. Hardner, M. B. Weissman, M. B. Salamon, and S. S. P. Parkin, Phys. Rev. B 48, 16156 (1993).

${ }^{33}$ S. Kogan, Electronic Noise and Fluctuations in Solids (Cambridge University press, Cambridge, England, 1996).

${ }^{34}$ L. Jiang, E. R. Nowak, P. E. Scott, J. Johnson, J. M. Slaughter, J. J. Sun, and R. W. Dave, Phys. Rev. B 69, 054407 (2004).

${ }^{35}$ G. Tatara and H. Kohno, Phys. Rev. Lett. 92, 086601 (2004).

${ }^{36}$ G. Tatara, H. Kohno, and J. Shibata, J. Phys. Soc. Jpn. 77, 031003 (2008).

${ }^{37}$ Y. Tserkovnyak, H. J. Skadsem, A. Brataas, and G. E. W. Bauer, Phys. Rev. B 74, 144405 (2006).

${ }^{38}$ H. Kohno, G. Tatara, and J. Shibata, J. Phys. Soc. Jpn. 75, 113706 (2006).

${ }^{39}$ R. A. Duine, A. S. Nunez, J. Sinova, and A. H. MacDonald, Phys. Rev. B 75, 214420 (2007).

${ }^{40}$ F. Piechon and A. Thiaville, Phys. Rev. B 75, 174414 (2007). 